\title{
Permanence and Periodic Solutions for a Two-Patch Impulsive Migration Periodic N-Species Lotka-Volterra Competitive System
}

\author{
Zijian Liu ${ }^{1,2}$ and Chenxue Yang ${ }^{3}$ \\ ${ }^{1}$ College of Mathematics and Statistics, Chongqing Jiaotong University, Chonging 400074, China \\ ${ }^{2}$ Department of Mathematics, Hangzhou Normal University, Hangzhou, Zhejiang 310036, China \\ ${ }^{3}$ School of Computer Science and Engineering, University of Electronic Science and Technology of China, \\ Chengdu 610054, China
}

Correspondence should be addressed to Zijian Liu; hbliuzijian@126.com

Received 21 August 2015; Accepted 12 November 2015

Academic Editor: Hassan A. El-Morshedy

Copyright (C) 2015 Z. Liu and C. Yang. This is an open access article distributed under the Creative Commons Attribution License, which permits unrestricted use, distribution, and reproduction in any medium, provided the original work is properly cited.

We study a two-patch impulsive migration periodic $N$-species Lotka-Volterra competitive system. Based on analysis method, inequality estimation, and Lyapunov function method, sufficient conditions for the permanence and existence of a unique globally stable positive periodic solution of the system are established. Some numerical examples are shown to verify our results and discuss the model further.

\section{Introduction}

Owing to natural enemy, severe competition, seasonal alternative, or deterioration of the patch environment, species dispersal (or migration) in two or more patches becomes one of the most prevalent phenomena of nature. Generally speaking, species dispersal is mainly concluded as the following three types: (i) dispersal occurs at every time and happens simultaneously between any two patches, that is, continuously bidirectional dispersal; (ii) dispersal occurs at some fixed time and happens simultaneously between any two patches, that is, impulsively bidirectional dispersal; (iii) dispersal shows itself as a total migration form, that is, impulsively unilateral diffusion (or migration).

Many empirical works and monographs on population dispersal system with type (i) have been done (see [1-6] and references cited therein). For example, in [3], Teng and $\mathrm{Lu}$ have investigated the following single-species nonautonomous dispersal model with delays:

$$
\begin{aligned}
& \frac{d x_{i}(t)}{d t}=x_{i}(t)\left(a_{i}(t)-b_{i}(t) x_{i}(t)-c_{i}(t) x_{i}(t-\tau(t))\right. \\
& \left.\quad-\int_{-\sigma}^{0} k_{i}(t, s) x_{i}(t+s) d s\right)
\end{aligned}
$$

$$
+\sum_{j=1}^{n} D_{i j}(t)\left(x_{j}(t)-x_{i}(t)\right)
$$

where $D_{i j}(t)$ represents the dispersal rate from patch $j$ to patch $i$ at time $t$ and the dispersal established in this model is continuous and bidirectional; that is, the dispersal occurs at every time and happens simultaneously between any two patches $i$ and $j$. In recent years, some population dynamical models with impulsively bidirectional dispersal have been proposed and studied (see [7-10] and references cited therein). For instance, in [7], the authors studied the following autonomous impulsive diffusion single species model:

$$
\begin{aligned}
& \frac{d Y_{1}(t)}{d t}=r_{1} Y_{1}(t) \ln \frac{k_{1}}{Y_{1}(t)} \\
& \frac{d Y_{2}(t)}{d t}=r_{2} Y_{2}(t) \ln \frac{k_{2}}{Y_{2}(t)}
\end{aligned}
$$




$$
\begin{aligned}
& \Delta Y_{1}=d_{1}\left(Y_{2}(t)-Y_{1}(t)\right), \\
& \Delta Y_{2}=d_{2}\left(Y_{1}(t)-Y_{2}(t)\right),
\end{aligned}
$$

$$
t=n \tau
$$

where $d_{i}(i=1,2)$ is the dispersal rate in the $i$ th patch. The pulse diffusion occurs at every $\tau$ period ( $\tau$ is a positive constant). Obviously, in this model, species $Y$ inhabits, respectively, two patches before the pulse appears; when the time at the pulse comes, species $Y$ in two patches disperses from one patch to another, that is, impulsively bidirectional dispersal.

However, in all of these investigated dispersal models considered so far, there are few papers to consider the total impulsive migration system, that is, impulsively unilateral diffusion (type (iii)) system. Practically, in the real ecological system, with seasonal alternative, some kinds of birds or vegetarians will migrate from cold patches (or food resource poor patches) to warm patches (or food resource rich patches) in search for a better habitat to inhabit or breed; fish will go back from ocean to their birthplace to spawn and so on. Obviously, this kind of diffusing behavior exists extensively in the real world. Therefore, it is a very basic problem to research this kind of impulsive migration systems. Zhang et al. in [11] studied a single species model with logistic growth and dissymmetric impulse dispersal and obtained some very general, weak conditions for the permanence, extinction of these systems, existence, uniqueness, and global stability of positive periodic solutions by using analysis based on the theory of discrete dynamical systems. In our previous work $[12,13]$, a two-patch impulsive diffusion periodic singlespecies logistic model (see [12]) and a two-patch prey impulsive diffusion periodic predator-prey model (see [13]) have been proposed and studied and some interesting results have been established, respectively. In this paper, we will continue our study on the two-patch impulsive diffusion model to a $N$-species competitive system.

Motivated by the above analysis, in this paper, we consider the following two-patch impulsive migration periodic $N$ species Lotka-Volterra competitive system:

$$
\begin{aligned}
\dot{x}_{i}(t) & =x_{i}(t)\left(a_{1 i}(t)-\sum_{j=1}^{n} b_{1 i j}(t) x_{j}(t)\right), \\
t \in\left[\tau_{2 k}, \tau_{2 k+1}\right), & \\
x_{i}\left(\tau_{2 k+1}\right) & =\left(1-D_{1 i}\right) x_{i}\left(\tau_{2 k+1}^{-}\right), \\
\dot{x}_{i}(t) & =x_{i}(t)\left(a_{2 i}(t)-\sum_{j=1}^{n} b_{2 i j}(t) x_{j}(t)\right), \\
x_{i}\left(\tau_{2 k+2}\right) & =\left(1-D_{2 i}\right) x_{i}\left(\tau_{2 k+2}^{-}\right), \\
i=1,2, \ldots, n, k=0,1,2, \ldots, & \left.t \tau_{2 k+1}, \tau_{2 k+2}\right),
\end{aligned}
$$

where $x_{i}$ is the population density of the $i$ th species; $a_{1 i}(t)$ and $a_{2 i}(t)$ represent the intrinsic growth rates of the $i$ th species in patch 1 and in patch 2, respectively; $b_{1 i i}(t)$ and $b_{2 i i}(t)$ denote the intraspecific competition coefficients of the $i$ th species in patch 1 and in patch 2, respectively; $b_{1 i j}(t)$ and $b_{2 i j}(t)(j \neq$ $i)$ are the interspecific competition coefficients between the $i$ th species and the $j$ th species in patch 1 and in patch 2 , respectively. The species migration occurs at every pulse time $\tau_{k+1}(k=0,1,2, \ldots)$, where $\tau_{0}=0, \tau_{1}<\tau_{2}<\cdots<\tau_{k}<\cdots$ is sequence of positive numbers with $\lim _{k \rightarrow \infty} \tau_{k}=+\infty$. We suppose that the system is composed of two patches. When $t \in\left[\tau_{2 k}, \tau_{2 k+1}\right)$, all the species live in patch 1; because of the change of the environment, the populations will migrate to patch 2 and the migration loss is $D_{1 i} x\left(\tau_{2 k+1}^{-}\right)(i=1,2, \ldots, n)$; then the populations will live in patch 2 during the period $t \in\left[\tau_{2 k+1}, \tau_{2 k+2}\right)$. When the environment changes again, all the populations will migrate back to the previous patch; here, the migration loss is $D_{2 i} x\left(\tau_{2 k+2}^{-}\right)$.

In this paper, we always assume the following:

$\left(\mathrm{H}_{1}\right)$ Functions $a_{1 i}(t), a_{2 i}(t), b_{1 i j}(t)$, and $b_{2 i j}(t)(i, j=$ $1,2, \ldots, n)$ are $T$-periodic continuous defined on $R_{+}=[0, \infty), b_{1 i j}(t) \geqslant 0$ and $b_{2 i j}(t) \geqslant 0$ for all $t \in R_{+}$ and $i, j=1,2, \ldots, n$.

$\left(\mathrm{H}_{2}\right)$ Impulsive time sequence $\left\{\tau_{k}\right\}$ satisfies $\tau_{k+2}=\tau_{k}+$ $T$ for all $k=0,1,2, \ldots$. Moreover, for all $i=$ $1,2, \ldots, n, D_{1 i} \in[0,1)$ and $D_{2 i} \in[0,1)$ are constants.

In addition, we assume that the investigated $N$ species always migrate between the two patches almost simultaneously. We will establish some sufficient conditions for the permanence, extinction, and existence of a unique globally asymptotically stable positive periodic solution of the system. The methods used in this paper are inequality estimation and Lyapunov functions which are introduced in work [14] "the permanence and global stability for nonautonomous $N$ species Lotka-Volterra competitive system with impulses."

The organization of this paper is as follows. In Section 2, as preliminary, an important lemma on the two-patch impulsive migration periodic single-species logistic model is introduced. In Section 3, sufficient conditions on the permanence and extinction of system (3) are established. In Section 4, conditions for the existence and global stability of the unique positive periodic solution are obtained. Finally, some examples and numerical simulations are proposed to illustrate the feasibility of our results and discuss the model further.

\section{Preliminaries}

In this section, as a preliminary we consider the following two-patch impulsive migration periodic single-species logistic system:

$$
\begin{aligned}
\dot{x}(t) & =x(t)\left(\alpha_{1}(t)-\beta_{1}(t) x(t)\right), \\
x\left(\tau_{2 k+1}\right) & =\left(1-D_{1}\right) x\left(\tau_{2 k+1}^{-}\right), \\
\dot{x}(t) & \left.=x(t)\left(\alpha_{2}(t)-\beta_{2 k}, \tau_{2 k+1}\right), x(t)\right), \\
& t \in\left[\tau_{2 k+1}, \tau_{2 k+2}\right), \\
x\left(\tau_{2 k+2}\right) & =\left(1-D_{2}\right) x\left(\tau_{2 k+2}^{-}\right), \quad k=0,1,2, \ldots,
\end{aligned}
$$


where $\alpha_{1}(t), \alpha_{2}(t), \beta_{1}(t)$, and $\beta_{2}(t)$ are $\omega$-periodic continuous functions defined on $R_{+}, \beta_{1}(t) \geqslant 0$, and $\beta_{2}(t) \geqslant 0$ for all $t \in R_{+}$ and impulsive time sequence $\left\{\tau_{k}\right\}$ satisfies $\tau_{k+2}=\tau_{k}+\omega$ for all $k=0,1,2, \ldots$ Moreover, $D_{1} \in[0,1)$ and $D_{2} \in[0,1)$ are constants. We have the following result.

Lemma 1. Let $x(t)$ be any positive solution of system (4).

(a) If system (4) satisfies

$$
\begin{aligned}
& \int_{0}^{\tau_{1}} \beta_{1}(t) d t+\int_{\tau_{1}}^{\omega} \beta_{2}(t) d t>0 \\
& \int_{0}^{\tau_{1}} \alpha_{1}(t) d t+\int_{\tau_{1}}^{\omega} \alpha_{2}(t) d t+\ln \left(1-D_{1}\right)+\ln \left(1-D_{2}\right) \\
& \quad>0
\end{aligned}
$$

then it has a unique globally attractively positive $\omega$-periodic solution $x^{*}(t)$; that is,

$$
\lim _{t \rightarrow \infty}\left(x(t)-x^{*}(t)\right)=0 .
$$

(b) If condition (6) is replaced by

$$
\begin{aligned}
& \int_{0}^{\tau_{1}} \alpha_{1}(t) d t+\int_{\tau_{1}}^{\omega} \alpha_{2}(t) d t+\ln \left(1-D_{1}\right)+\ln \left(1-D_{2}\right) \\
& \quad \leqslant 0
\end{aligned}
$$

and condition (5) is retained, then

$$
\lim _{t \rightarrow \infty} x(t)=0
$$

Proof. Due to the fact that the population dispersal is only restricted in two patches and shows itself as aggregate migration, we can rewrite system (4) as follows:

$$
\begin{aligned}
\dot{x}(t)= & \frac{(-1)^{k}+1}{2} x(t)\left(\alpha_{1}(t)-\beta_{1}(t) x(t)\right) \\
& +\frac{(-1)^{k+1}+1}{2} x(t)\left(\alpha_{2}(t)-\beta_{2}(t) x(t)\right), \\
& t \in\left[\tau_{k}, \tau_{k+1}\right), \\
x\left(\tau_{k+1}\right)= & \frac{(-1)^{k}+1}{2}\left(1-D_{1}\right) x\left(\tau_{k+1}^{-}\right)
\end{aligned}
$$

$$
\begin{array}{r}
+\frac{(-1)^{k+1}+1}{2}\left(1-D_{2}\right) \times\left(\tau_{k+1}^{-}\right) \\
\quad k=0,1,2, \ldots .
\end{array}
$$

In order to prove proposition (a), firstly, we prove the permanence of system (4); that is, there exist two positive constants $m$ and $M$ such that for any positive solution $x(t)$ of system (4) we always have

$$
m<\liminf _{t \rightarrow \infty} x(t) \leqslant \limsup _{t \rightarrow \infty} x(t)<M .
$$

From conditions (5) and (6), there are positive constants $e_{1}, e_{2}$, and $\delta$ such that

$$
\begin{aligned}
& \int_{0}^{\tau_{1}}\left(\alpha_{1}(t)-\beta_{1}(t) e_{1}\right) d t+\int_{\tau_{1}}^{\omega}\left(\alpha_{2}(t)-\beta_{2}(t) e_{1}\right) d t \\
& \quad+\ln \left(1-D_{1}\right)+\ln \left(1-D_{2}\right)<-\delta, \\
& \int_{0}^{\tau_{1}}\left(\alpha_{1}(t)-\beta_{1}(t) e_{2}\right) d t+\int_{\tau_{1}}^{\omega}\left(\alpha_{2}(t)-\beta_{2}(t) e_{2}\right) d t \\
& \quad+\ln \left(1-D_{1}\right)+\ln \left(1-D_{2}\right)>\delta .
\end{aligned}
$$

We first of all prove that there is a constant $M>0$ such that

$$
\limsup _{t \rightarrow \infty} x(t)<M
$$

for any positive solution of system (4). In fact, for any positive solution of system (4), we only need to consider the following three cases.

Case 1. There is $t_{0} \geqslant 0$ such that $x(t) \geqslant e_{1}$ for all $t \geqslant t_{0}$.

Case 2. There is $t_{0} \geqslant 0$ such that $x(t) \leqslant e_{1}$ for all $t \geqslant t_{0}$.

Case 3. $x(t)$ is oscillatory about $e_{1}$ for all $t \geqslant 0$.

We first consider Case 1 . Since $x(t) \geqslant e_{1}$ for all $t \geqslant t_{0}$, then for $t=t_{0}+l \omega$, where $l$ is any positive integer, integrating system (10) from $t_{0}$ to $t$, by (12), we have

$$
\begin{aligned}
& x(t)=x\left(t_{0}\right) \exp \left(\int_{t_{0}}^{t}\left(\frac{(-1)^{k}+1}{2}\left(\alpha_{1}(s)-\beta_{1}(s) x(s)\right)+\frac{(-1)^{k+1}+1}{2}\left(\alpha_{2}(s)-\beta_{2}(s) x(s)\right)\right) d s\right. \\
& \left.\quad+\sum_{t_{0} \leqslant \tau_{k}<t} \ln \left(\frac{(-1)^{k}+1}{2}\left(1-D_{1}\right)+\frac{(-1)^{k+1}+1}{2}\left(1-D_{2}\right)\right)\right) \leqslant x\left(t_{0}\right) \\
& \quad \cdot \exp \left(l\left(\int_{0}^{\omega}\left(\frac{(-1)^{k}+1}{2}\left(\alpha_{1}(s)-\beta_{1}(s) e_{1}\right)+\frac{(-1)^{k+1}+1}{2}\left(\alpha_{2}(s)-\beta_{2}(s) e_{1}\right)\right) d s+\ln \left(1-D_{1}\right)+\ln \left(1-D_{2}\right)\right)\right) \\
& \quad=x\left(t_{0}\right) \exp \left(l\left(\int_{0}^{\tau_{1}}\left(\alpha_{1}(s)-\beta_{1}(s) e_{1}\right) d s+\int_{\tau_{1}}^{\omega}\left(\alpha_{2}(s)-\beta_{2}(s) e_{1}\right) d s+\ln \left(1-D_{1}\right)+\ln \left(1-D_{2}\right)\right)\right) \leqslant x\left(t_{0}\right) \exp (-l \delta) .
\end{aligned}
$$


Hence, $x(t) \rightarrow 0$ as $l \rightarrow \infty$, which leads to a contradiction.

Next, we consider Case 3. Obviously, there is $t_{1} \geqslant 0$ such that $x\left(t_{1}\right)<e_{1}$. Then we prove that, for all $t \geqslant t_{1}$,

$$
x(t) \leqslant e_{1} \exp \left(\alpha_{0} \omega\right),
$$

where $\alpha_{0}=\max _{t \in[0, \omega]}\left\{\left|\alpha_{1}(t)\right|+\left|\alpha_{2}(t)\right|+\left(\beta_{1}(t)+\beta_{2}(t)\right) e_{1}\right\}$. If (16) is not true, then there is $t_{2}>t_{1}$ such that

$$
x\left(t_{2}\right)>e_{1} \exp \left(\alpha_{0} \omega\right) .
$$

Furthermore, there exists $t_{3} \in\left(t_{1}, t_{2}\right]$ such that $x\left(t_{3}\right)=e_{1}$ and $x(t) \geqslant e_{1}$ for all $t \in\left[t_{3}, t_{2}\right]$. Taking an integer $p \geqslant 0$ such that $t_{2} \in\left[t_{3}+p \omega, t_{3}+(p+1) \omega\right)$, then for all $t \in\left[t_{3}, t_{2}\right]$ we have

$$
\dot{x}(t) \leqslant \frac{(-1)^{k}+1}{2} x(t)\left(\alpha_{1}(t)-\beta_{1}(t) e_{1}\right)
$$

$$
\begin{gathered}
+\frac{(-1)^{k+1}+1}{2} x(t)\left(\alpha_{2}(t)-\beta_{2}(t) e_{1}\right), \\
t \in\left[\tau_{k}, \tau_{k+1}\right), \\
x\left(\tau_{k+1}\right)=\frac{(-1)^{k}+1}{2}\left(1-D_{1}\right) x\left(\tau_{k+1}^{-}\right) \\
+\frac{(-1)^{k+1}+1}{2}\left(1-D_{2}\right) x\left(\tau_{k+1}^{-}\right), \\
\quad k=0,1,2, \ldots,
\end{gathered}
$$

and integrating this inequality from $t_{3}$ to $t_{2}$ we have

$$
\begin{aligned}
& x\left(t_{2}\right) \\
& \leqslant x\left(t_{3}\right) \exp \left(\int_{t_{3}}^{t_{2}}\left(\frac{(-1)^{k}+1}{2}\left(\alpha_{1}(s)-\beta_{1}(s) e_{1}\right)+\frac{(-1)^{k+1}+1}{2}\left(\alpha_{2}(s)-\beta_{2}(s) e_{1}\right)\right) d s+p\left(\ln \left(1-D_{1}\right)+\ln \left(1-D_{2}\right)\right)\right) \\
& \leqslant e_{1} \exp (-p \delta) \exp \left(\int_{t_{3}+p \omega}^{t_{2}}\left(\frac{(-1)^{k}+1}{2}\left(\alpha_{1}(s)-\beta_{1}(s) e_{1}\right)+\frac{(-1)^{k+1}+1}{2}\left(\alpha_{2}(s)-\beta_{2}(s) e_{1}\right)\right) d s\right) \leqslant e_{1} \exp \left(\alpha_{0} \omega\right),
\end{aligned}
$$

which contradicts with (17). This proves that (16) holds.

Lastly, if Case 2 holds, then we directly have

$$
x(t) \leqslant e_{1} \exp \left(\alpha_{0} \omega\right) \quad \forall t \geqslant t_{1} .
$$

Choose constant $M=e_{1} \exp \left(\alpha_{0} \omega\right)+1$; then we see that (14) holds.

By a similar argument as in the proof of (14) we can prove that there is a constant $m>0$ such that

$$
\liminf _{t \rightarrow \infty} x(t)>m
$$

for any positive solution $x(t)$ of system (4). Conclusion (11) is proved.

Now, we prove proposition (a). Let $x(t)$ and $x^{*}(t)$ be any two positive solutions of system (4). It follows from (11) that there are positive constants $A$ and $B$ such that

$$
\begin{aligned}
A & \leqslant x(t), \\
x^{*}(t) & \leqslant B,
\end{aligned}
$$

$$
\forall t \geqslant 0 \text {. }
$$

Choose Lyapunov function as follows:

$$
V(t)=\left|\ln x(t)-\ln x^{*}(t)\right| .
$$

For any $k=0,1,2, \ldots$, we have

$$
\begin{gathered}
V\left(\tau_{k+1}\right)=\mid \ln \left(\frac{(-1)^{k}+1}{2}\left(1-D_{1}\right)\right. \\
\left.+\frac{(-1)^{k+1}+1}{2}\left(1-D_{2}\right)\right) x\left(\tau_{k+1}^{-}\right)
\end{gathered}
$$

$$
\begin{aligned}
& -\ln \left(\frac{(-1)^{k}+1}{2}\left(1-D_{1}\right)+\frac{(-1)^{k+1}+1}{2}(1\right. \\
& \left.\left.-D_{2}\right)\right) x^{*}\left(\tau_{k+1}^{-}\right) \mid=V\left(\tau_{k+1}^{-}\right) .
\end{aligned}
$$

Hence, $V(t)$ is continuous for all $t \in R_{+}$and from the MeanValue Theorem we can obtain

$$
\frac{1}{B}\left|x(t)-x^{*}(t)\right| \leqslant V(t) \leqslant \frac{1}{A}\left|x(t)-x^{*}(t)\right| .
$$

Calculating the upper right derivative of $V(t)$, then from (25) we obtain

$$
\begin{aligned}
& D^{+} V(t)=\operatorname{sign}\left(x(t)-x^{*}(t)\right)\left(\frac{\dot{x}(t)}{x(t)}-\frac{\dot{x}^{*}(t)}{x^{*}(t)}\right) \\
& =\left(\frac{(-1)^{k}+1}{2}\left(-\beta_{1}(t)\right)+\frac{(-1)^{k+1}+1}{2}\left(-\beta_{2}(t)\right)\right) \\
& \cdot\left|x(t)-x^{*}(t)\right| \\
& \leqslant-\left(\frac{(-1)^{k}+1}{2} \beta_{1}(t)+\frac{(-1)^{k+1}+1}{2} \beta_{2}(t)\right) \\
& \cdot A V(t), \quad t \neq \tau_{k+1}, \quad k=0,1,2, \ldots
\end{aligned}
$$


From this, we further have, for any $t=p \omega+\gamma$, where $p \geqslant 0$ is an integer and $0 \leqslant \gamma<\omega$ is a constant,

$$
\begin{aligned}
& V(t) \leqslant V(0) \\
& \cdot \exp \left(-A\left(\int_{0}^{t}\left(\frac{(-1)^{k}+1}{2} \beta_{1}(s)+\frac{(-1)^{k+1}+1}{2} \beta_{2}(s)\right) d s\right)\right) \\
& =V(0) \exp \left(-A\left(\int_{0}^{p \omega}+\int_{p \omega}^{t}\right)\right. \\
& \left.\cdot\left(\frac{(-1)^{k}+1}{2} \beta_{1}(s)+\frac{(-1)^{k+1}+1}{2} \beta_{2}(s)\right) d s\right) \leqslant V(0) \\
& \cdot \exp \left(-A p\left(\int_{0}^{\tau_{1}} \beta_{1}(s) d s+\int_{\tau_{1}}^{\omega} \beta_{2}(s) d s\right)\right) \rightarrow 0,
\end{aligned}
$$

$$
p \longrightarrow \infty \text {. }
$$

Hence, $V(t) \rightarrow 0$ as $t \rightarrow \infty$. Further from (25) we obtain

$$
\lim _{t \rightarrow \infty}\left(x(t)-x^{*}(t)\right)=0 .
$$

Lastly, we prove that system (4) has a unique positive $\omega$-periodic solution. Consider the sequence $x^{*}\left(m \omega, x_{0}^{*}\right)$. It is obviously bounded in the interval $[A, B]$ for all $m=0,1, \ldots$. Let $\bar{x}^{*}$ be a limit point of this sequence, $\bar{x}^{*}=\lim _{n \rightarrow \infty} x^{*}\left(m_{n} \omega, x_{0}^{*}\right)$. Then $x^{*}\left(\omega, \bar{x}^{*}\right)=\bar{x}^{*}$. Indeed, since $x^{*}\left(\omega, x^{*}\left(m_{n} \omega, x_{0}^{*}\right)\right)=x^{*}\left(m_{n} \omega, x^{*}\left(\omega, x_{0}^{*}\right)\right)$ and $x^{*}\left(m_{n} \omega, x^{*}\left(\omega, x_{0}^{*}\right)\right)-x^{*}\left(m_{n} \omega, x_{0}^{*}\right) \rightarrow 0$ as $m_{n} \rightarrow \infty$, we get

$$
\begin{aligned}
&\left\|x^{*}\left(\omega, \bar{x}^{*}\right)-\bar{x}^{*}\right\|_{[A, B]} \\
& \leqslant\left\|x^{*}\left(\omega, \bar{x}^{*}\right)-x^{*}\left(\omega, x^{*}\left(m_{n} \omega, x_{0}^{*}\right)\right)\right\|_{[A, B]} \\
&+\left\|x^{*}\left(\omega, x^{*}\left(m_{n} \omega, x_{0}^{*}\right)\right)-x^{*}\left(m_{n} \omega, x_{0}^{*}\right)\right\|_{[A, B]} \\
&+\left\|x^{*}\left(m_{n} \omega, x_{0}^{*}\right)-\bar{x}^{*}\right\|_{[A, B]} \longrightarrow 0, \quad n \longrightarrow \infty .
\end{aligned}
$$

The sequence $x^{*}\left(m \omega, x_{0}^{*}\right), m=1,2, \ldots$, has a unique limit point. On the contrary, let the sequence have two limit points $\bar{x}^{*}=\lim _{n \rightarrow \infty} x^{*}\left(m_{n} \omega, x_{0}^{*}\right)$ and $\tilde{x}^{*}=\lim _{n \rightarrow \infty} x^{*}\left(m_{n} \omega, x_{0}^{*}\right)$. Then, taking into account (28) and $\tilde{x}^{*}=x^{*}\left(m_{n} \omega, \tilde{x}^{*}\right)$, we have

$$
\begin{aligned}
\left\|\bar{x}^{*}-\tilde{x}^{*}\right\|_{[A, B]} \leqslant\left\|\bar{x}^{*}-x^{*}\left(m_{n} \omega, x_{0}^{*}\right)\right\|_{[A, B]} & \\
+\left\|x^{*}\left(m_{n} \omega, x_{0}^{*}\right)-\tilde{x}^{*}\right\|_{[A, B]} & \longrightarrow 0, \\
n & \longrightarrow \infty,
\end{aligned}
$$

and hence $\bar{x}^{*}=\tilde{x}^{*}$. The solution $x^{*}\left(t, \bar{x}^{*}\right)$ is the unique periodic solution of system (4). By (28), it is globally attractive. This completes the proof of proposition (a).

Now we prove proposition (b). From (5) and (8), for any constant $\eta>0$, there is a positive constant $\delta_{0}$ such that

$$
\begin{gathered}
\int_{0}^{\tau_{1}}\left(\alpha_{1}(t)-\beta_{1}(t) \eta\right) d t+\int_{\tau_{1}}^{\omega}\left(\alpha_{2}(t)-\beta_{2}(t) \eta\right) d t \\
+\ln \left(1-D_{1}\right)+\ln \left(1-D_{2}\right)<-\delta_{0} .
\end{gathered}
$$

From this, a similar argument as in the proof of (14), we can obtain

$$
x(t) \leqslant \eta \exp \left(\alpha_{0} \omega\right)
$$

for all $t$ large enough. Finally, from the arbitrariness of $\eta$, we obtain $x(t) \rightarrow 0$ as $t \rightarrow \infty$. Lemma 1 is proved.

Remark 2. In [12], to prove the globally attractively positive $\omega$-periodic solution and the extinction of system (4), we required conditions $\beta_{1}(t)>0$ and $\beta_{2}(t)>0$ for all $t \epsilon$ $R_{+}$besides conditions (6) and (8). However, we improve the conditions $\beta_{1}(t)>0$ and $\beta_{2}(t)>0$ for all $t \in R_{+}$to $\int_{0}^{\tau_{1}} \beta_{1}(t) d t+\int_{\tau_{1}}^{\omega} \beta_{2}(t) d t>0$ (condition (5)) in Lemma 1, which is superior to conditions given in [12].

\section{Permanence and Extinction}

We first discuss the permanence of all species of system (3). A similar analysis as system (4), system (3) can also be written as follows:

$$
\begin{aligned}
& \dot{x}_{i}(t) \\
& =\frac{(-1)^{k}+1}{2} x_{i}(t)\left(a_{1 i}(t)-\sum_{j=1}^{n} b_{1 i j}(t) x_{j}(t)\right) \\
& \quad+\frac{(-1)^{k+1}+1}{2} x_{i}(t)\left(a_{2 i}(t)-\sum_{j=1}^{n} b_{2 i j}(t) x_{j}(t)\right), \\
& x_{i}\left(\tau_{k+1}\right) \\
& =\frac{(-1)^{k}+1}{2}\left(1-D_{1 i}\right) x_{i}\left(\tau_{k+1}^{-}\right) \\
& \quad+\frac{(-1)^{k+1}+1}{2}\left(1-\tau_{2 i}\right) x_{i}\left(\tau_{k+1}^{-}\right), \\
& \quad i=1,2, \ldots, n, k=0,1,2, \ldots
\end{aligned}
$$

For each $i=1,2, \ldots, n$, we consider the following two-patch impulsive migration systems as the subsystems of system (3):

$$
\begin{gathered}
\dot{x}_{i}(t)=x_{i}(t)\left(a_{1 i}(t)-b_{1 i i}(t) x_{i}(t)\right), \\
t \in\left[\tau_{2 k}, \tau_{2 k+1}\right), \\
x_{i}\left(\tau_{2 k+1}\right)=\left(1-D_{1 i}\right) x_{i}\left(\tau_{2 k+1}^{-}\right), \\
\dot{x}_{i}(t)=x_{i}(t)\left(a_{2 i}(t)-b_{2 i i}(t) x_{i}(t)\right), \\
t \in\left[\tau_{2 k+1}, \tau_{2 k+2}\right), \\
x_{i}\left(\tau_{2 k+2}\right)=\left(1-D_{2 i}\right) x_{i}\left(\tau_{2 k+2}^{-}\right), \quad k=0,1,2, \ldots .
\end{gathered}
$$

On the permanence of all species $x_{i}(i=1,2, \ldots, n)$ for system (3) we have the following result. 
Theorem 3. Assume that conditions $\left(H_{1}\right)$ and $\left(H_{2}\right)$ hold. Moreover, if

$$
\begin{aligned}
& \int_{0}^{\tau_{1}} b_{1 i i}(t) d t+\int_{\tau_{1}}^{T} b_{2 i i}(t) d t>0 \\
& \int_{0}^{\tau_{1}}\left(a_{1 i}(t)-\sum_{j \neq i}^{n} b_{1 i j}(t) x_{j}^{*}(t)\right) d t \\
& \quad+\int_{\tau_{1}}^{T}\left(a_{2 i}(t)-\sum_{j \neq i}^{n} b_{2 i j}(t) x_{j}^{*}(t)\right) d t+\ln \left(1-D_{1 i}\right) \\
& \quad+\ln \left(1-D_{2 i}\right)>0
\end{aligned}
$$

then system (3) is permanent; that is, there are constants $m>0$ and $M>0$ such that

$$
\begin{array}{r}
m<\liminf _{t \rightarrow \infty} x_{i}(t) \leqslant \limsup _{t \rightarrow \infty} x_{i}(t)<M, \\
\qquad i=1,2, \ldots, n
\end{array}
$$

for any positive solution $x(t)=\left(x_{1}(t), x_{2}(t), \ldots, x_{n}(t)\right)$ of system (3), where $x_{i}^{*}(t)(i=1,2, \ldots, n)$ is the globally attractively positive T-periodic solution of system (34).

Proof. From condition (36) we directly have

$$
\begin{gathered}
\int_{0}^{\tau_{1}} a_{1 i}(t) d t+\int_{\tau_{1}}^{T} a_{2 i}(t) d t+\ln \left(1-D_{1 i}\right) \\
+\ln \left(1-D_{2 i}\right)>0,
\end{gathered}
$$

and by Lemma $1(\mathrm{a})$ we can obtain that $x_{i}^{*}(t)$ defined in Theorem 3 is existent and globally attractive. Therefore, for any positive solution $x_{i}(t)$ of system (34) and any constant $\varepsilon>0$, there exists $T_{\varepsilon}>0$ such that

$$
x_{i}(t) \leqslant x_{i}^{*}(t)+\varepsilon \quad \forall t \geqslant T_{\varepsilon}, i=1,2, \ldots, n .
$$

We firstly prove the ultimately upper boundedness of system (3). From conditions (35) and (36), there are constants $\varepsilon_{0}>0$ small enough such that

$$
\begin{aligned}
& \int_{0}^{\tau_{1}}\left(a_{1 i}(t)-b_{1 i i}(t) \varepsilon_{0}-\sum_{j \neq i}^{n} b_{1 i j}(t)\left(x_{j}^{*}(t)+\varepsilon_{0}\right)\right) d t \\
& +\int_{\tau_{1}}^{T}\left(a_{2 i}(t)-b_{2 i i}(t) \varepsilon_{0}\right.
\end{aligned}
$$

$$
\begin{aligned}
& \left.-\sum_{j \neq i}^{n} b_{2 i j}(t)\left(x_{j}^{*}(t)+\varepsilon_{0}\right)\right) d t+\ln \left(1-D_{1 i}\right) \\
& +\ln \left(1-D_{2 i}\right)>\varepsilon_{0}
\end{aligned}
$$

for each $i=1,2, \ldots, n$. Let $x(t)=\left(x_{1}(t), x_{2}(t), \ldots, x_{n}(t)\right)$ be any positive solution of system (3). Since

$$
\begin{gathered}
\dot{x}_{i}(t) \leqslant x_{i}(t)\left(a_{1 i}(t)-b_{1 i i}(t) x_{i}(t)\right), \\
t \in\left[\tau_{2 k}, \tau_{2 k+1}\right), \\
x_{i}\left(\tau_{2 k+1}\right)=\left(1-D_{1 i}\right) x_{i}\left(\tau_{2 k+1}^{-}\right), \\
\dot{x}_{i}(t) \leqslant x_{i}(t)\left(a_{2 i}(t)-b_{2 i i}(t) x_{i}(t)\right), \\
t \in\left[\tau_{2 k+1}, \tau_{2 k+2}\right), \\
x_{i}\left(\tau_{2 k+2}\right)=\left(1-D_{2 i}\right) x_{i}\left(\tau_{2 k+2}^{-}\right), \quad k=0,1,2, \ldots,
\end{gathered}
$$

by the comparison theorem of impulsive differential equations, we obtain

$$
x_{i}(t) \leqslant u_{i}(t) \quad \forall t \geqslant 0,
$$

where $u_{i}(t)$ is the positive solution of system (34) with initial condition $u_{i}(0)=x_{i}(0)$. By taking $\varepsilon=\varepsilon_{0}$ in (39), we can obtain that

$$
x_{i}(t) \leqslant x_{i}^{*}(t)+\varepsilon_{0} \quad \forall t \geqslant T_{\varepsilon_{0}}, i=1,2, \ldots, n .
$$

Choose a constant $M=\max _{t \in[0, T]}\left\{x_{i}^{*}(t)+\varepsilon_{0}: i=1,2, \ldots, n\right\}$; then $M$ is independent of any positive solution of system (3). Obviously, we have $x_{i}(t) \leqslant M$ for all $t \geqslant T_{\varepsilon_{0}}$ and $i=1,2, \ldots, n$.

Next, we prove that there is a constant $m>0$ such that

$$
\liminf _{t \rightarrow \infty} x_{i}(t)>m, \quad i=1,2, \ldots, n
$$

We only need to consider the following three cases for each $i=1,2, \ldots, n$.

Case 1. There is $t_{4} \geqslant T_{\varepsilon_{0}}$ such that $x_{i}(t) \leqslant \varepsilon_{0}$ for all $t \geqslant t_{4}$.

Case 2. There is $t_{4} \geqslant T_{\varepsilon_{0}}$ such that $x_{i}(t) \geqslant \varepsilon_{0}$ for all $t \geqslant t_{4}$.

Case 3. $x_{i}(t)$ is oscillatory about $\varepsilon_{0}$ for all $t \geqslant T_{\varepsilon_{0}}$. 
For Case 1 , since $x_{i}(t) \leqslant \varepsilon_{0}$ for all $t \geqslant t_{4}$, then let $t=t_{4}+l T$, where $l$ is any positive integer; integrating system (33) from $t_{4}$ to $t$, by (40) and (43) we have

$$
\begin{aligned}
& x_{i}(t)=x_{i}\left(t_{4}\right) \\
& \quad \cdot \exp \left(\int_{t_{4}}^{t}\left(\frac{(-1)^{k}+1}{2}\left(a_{1 i}(s)-b_{1 i i}(s) x_{i}(s)-\sum_{j \neq i}^{n} b_{1 i j}(s) x_{j}(s)\right)+\frac{(-1)^{k+1}+1}{2}\left(a_{2 i}(s)-b_{2 i i}(s) x_{i}(s)-\sum_{j \neq i}^{n} b_{2 i j}(s) x_{j}(s)\right)\right) d s\right. \\
& \left.\quad+\sum_{t_{4} \leqslant \tau_{k}<t} \ln \left(\frac{(-1)^{k}+1}{2}\left(1-D_{1 i}\right)+\frac{(-1)^{k+1}+1}{2}\left(1-D_{2 i}\right)\right)\right) \geqslant x_{i}\left(t_{4}\right) \\
& \quad \cdot \exp \left(l \left(\int_{0}^{\tau_{1}}\left(a_{1 i}(s)-b_{1 i i}(s) \varepsilon_{0}-\sum_{j \neq i}^{n} b_{1 i j}(s)\left(x_{j}^{*}(s)+\varepsilon_{0}\right)\right) d s+\int_{\tau_{1}}^{T}\left(a_{2 i}(s)-b_{2 i i}(s) \varepsilon_{0}-\sum_{j \neq i}^{n} b_{2 i j}(s)\left(x_{j}^{*}(s)+\varepsilon_{0}\right)\right) d s\right.\right. \\
& \left.\left.\quad+\ln \left(1-D_{1 i}\right)+\ln \left(1-D_{2 i}\right)\right)\right) \geqslant x_{i}\left(t_{4}\right) \exp \left(l \varepsilon_{0}\right) .
\end{aligned}
$$

Hence, $x_{i}(t) \rightarrow 0$ as $l \rightarrow \infty$, which leads to a contradiction.

For Case 3, obviously, there is $t_{5} \geqslant T_{\varepsilon_{0}}$ such that $x_{i}\left(t_{5}\right)>$ $\varepsilon_{0}$. Then we prove that, for all $t \geqslant t_{5}$,

$$
x_{i}(t) \geqslant \varepsilon_{0} \exp \left(-\gamma_{0} T\right)\left(1-D_{1 i}\right)\left(1-D_{2 i}\right)
$$

where

$$
\begin{aligned}
\gamma_{0} & =\max _{t \in[0, T]}\left\{\left|a_{1 i}(t)\right|+\left|a_{2 i}(t)\right|+\left(b_{1 i i}(t)+b_{2 i i}(t)\right) \varepsilon_{0}\right. \\
& \left.+\sum_{j \neq i}^{n} b_{1 i j}(t)\left(x_{j}^{*}(t)+\varepsilon_{0}\right)+\sum_{j \neq i}^{n} b_{2 i j}(t)\left(x_{j}^{*}(t)+\varepsilon_{0}\right)\right\} .
\end{aligned}
$$

If (46) is not true, then there is $t_{6}>t_{5}$ such that

$$
x_{i}\left(t_{6}\right)<\varepsilon_{0} \exp \left(-\gamma_{0} T\right)\left(1-D_{1 i}\right)\left(1-D_{2 i}\right) \text {. }
$$

Moreover, there exists $t_{7} \in\left(t_{5}, t_{6}\right]$ such that

$$
\begin{aligned}
& x_{i}\left(t_{7}\right) \leqslant \varepsilon_{0}, \\
& x_{i}\left(t_{7}^{-}\right) \geqslant \varepsilon_{0}, \\
& x_{i}(t) \leqslant \varepsilon_{0} \quad \forall t \in\left[t_{7}, t_{6}\right] .
\end{aligned}
$$

If $t_{6}=t_{7}, t_{6}$ must be an impulsive time. Then there exists a positive integer $k$ such that $t_{6}=\tau_{2 k}$ or $t_{6}=\tau_{2 k+1}$; thus we have

$$
x_{i}\left(t_{6}\right)= \begin{cases}x_{i}\left(t_{6}^{-}\right)\left(1-D_{2 i}\right), & t=\tau_{2 k}, \\ x_{i}\left(t_{6}^{-}\right)\left(1-D_{1 i}\right), & t=\tau_{2 k+1} .\end{cases}
$$

From this we can obtain

$$
\begin{aligned}
x_{i}\left(t_{6}\right) & \geqslant \varepsilon_{0}\left(1-D_{1 i}\right)\left(1-D_{2 i}\right) \\
& >\varepsilon_{0} \exp \left(-\gamma_{0} T\right)\left(1-D_{1 i}\right)\left(1-D_{2 i}\right),
\end{aligned}
$$

which contradicts with (48). If $t_{6}>t_{7}$, we can choose an integer $p \geqslant 0$ such that $t_{6} \in\left[t_{7}+p T, t_{7}+(p+1) T\right)$; then we have for all $t \in\left[t_{7}, t_{6}\right]$

$$
\dot{x}_{i}(t) \geqslant \frac{(-1)^{k}+1}{2} x_{i}(t)
$$

$$
\cdot\left(a_{1 i}(t)-b_{1 i i}(t) \varepsilon_{0}-\sum_{j \neq i}^{n} b_{1 i j}(t)\left(x_{j}^{*}(t)+\varepsilon_{0}\right)\right)
$$$$
+\frac{(-1)^{k+1}+1}{2} x_{i}(t)
$$$$
\cdot\left(a_{2 i}(t)-b_{2 i i}(t) \varepsilon_{0}-\sum_{j \neq i}^{n} b_{2 i j}(t)\left(x_{j}^{*}(t)+\varepsilon_{0}\right)\right) \text {, }
$$

$$
t \in\left[\tau_{k}, \tau_{k+1}\right),
$$

$$
\begin{aligned}
& x_{i}\left(\tau_{k+1}\right)=\frac{(-1)^{k}+1}{2}\left(1-D_{1 i}\right) x_{i}\left(\tau_{k+1}^{-}\right) \\
& +\frac{(-1)^{k+1}+1}{2}\left(1-D_{2 i}\right) x_{i}\left(\tau_{k+1}^{-}\right), \quad k=0,1,2, \ldots,
\end{aligned}
$$


and integrating this inequality from $t_{7}$ to $t_{6}$ we have

$$
\begin{aligned}
& x_{i}\left(t_{6}\right) \geqslant x_{i}\left(t_{7}^{-}\right) \\
& \cdot \exp \left(\int_{t_{7}}^{t_{6}}\left(\frac{(-1)^{k}+1}{2}\left(a_{1 i}(s)-b_{1 i i}(s) \varepsilon_{0}-\sum_{j \neq i}^{n} b_{1 i j}(s)\left(x_{j}^{*}(s)+\varepsilon_{0}\right)\right)+\frac{(-1)^{k+1}+1}{2}\left(a_{2 i}(s)-b_{2 i i}(s) \varepsilon_{0}-\sum_{j \neq i}^{n} b_{2 i j}(s)\left(x_{j}^{*}(s) \varepsilon_{0}\right)\right)\right) d s\right) \\
& \cdot\left(\left(1-D_{1 i}\right)\left(1-D_{2 i}\right)\right)^{p+1} \geqslant \varepsilon_{0} \exp \left(p \varepsilon_{0}\right) \\
& \quad \cdot \exp \left(\int_{t_{7}+p T}^{t_{6}}\left(\frac{(-1)^{k}+1}{2}\left(a_{1 i}(s)-b_{1 i i}(s) \varepsilon_{0}-\sum_{j \neq i}^{n} b_{1 i j}(s)\left(x_{j}^{*}(s)+\varepsilon_{0}\right)\right)+\frac{(-1)^{k+1}+1}{2}\left(a_{2 i}(s)-b_{2 i i}(s) \varepsilon_{0}-\sum_{j \neq i}^{n} b_{2 i j}(s)\left(x_{j}^{*}(s) \varepsilon_{0}\right)\right)\right) d s\right) \\
& \cdot\left(1-D_{1 i}\right)\left(1-D_{2 i}\right) \geqslant \varepsilon_{0} \exp \left(-\gamma_{0} T\right)\left(1-D_{1 i}\right)\left(1-D_{2 i}\right),
\end{aligned}
$$

which contradicts with (48) too. This proves that (46) holds. Lastly, if Case 2 holds, then we directly have

$$
\begin{aligned}
& x_{i}(t) \geqslant \varepsilon_{0} \geqslant \varepsilon_{0} \exp \left(-\gamma_{0} T\right)\left(1-D_{1 i}\right)\left(1-D_{2 i}\right) \\
& \forall t \geqslant t_{4} .
\end{aligned}
$$

Let constant $m=\min _{1 \leqslant i \leqslant n}\left\{\varepsilon_{0} \exp \left(-\gamma_{0} T-1\right)\left(1-D_{1 i}\right)\left(1-D_{2 i}\right)\right\}$. Then $m$ is independent of any positive solution of system (3) and we finally have

$$
\liminf _{t \rightarrow \infty} x_{i}(t)>m, \quad i=1,2, \ldots, n
$$

This completes the proof of Theorem 3.

Next, we study the extinction of all species $x_{i}$ for system (3); we have the following result.

Theorem 4. Assume that conditions $\left(\mathrm{H}_{1}\right)$ and $\left(\mathrm{H}_{2}\right)$ hold. Moreover, if

$$
\begin{aligned}
& \int_{0}^{\tau_{1}} b_{1 i i}(t) d t+\int_{\tau_{1}}^{T} b_{2 i i}(t) d t>0 \\
& \int_{0}^{\tau_{1}} a_{1 i}(t) d t+\int_{\tau_{1}}^{T} a_{2 i}(t) d t+\ln \left(1-D_{1 i}\right) \\
& \quad+\ln \left(1-D_{2 i}\right) \leqslant 0,
\end{aligned}
$$

then all species of system (3) are extinct; that is,

$$
\lim _{t \rightarrow \infty} x_{i}(t)=0, \quad i=1,2, \ldots, n
$$

for any positive solution $x(t)=\left(x_{1}(t), x_{2}(t), \ldots, x_{n}(t)\right)$ of system (3).

Proof. From system (3) we directly have

$$
\begin{aligned}
\dot{x}_{i}(t) \leqslant x_{i}(t)\left(a_{1 i}(t)-b_{1 i i}(t) x_{i}(t)\right) & \\
t & \in\left[\tau_{2 k}, \tau_{2 k+1}\right),
\end{aligned}
$$

$$
\begin{aligned}
x_{i}\left(\tau_{2 k+1}\right) & =\left(1-D_{1 i}\right) x_{i}\left(\tau_{2 k+1}^{-}\right), \\
\dot{x}_{i}(t) \leqslant x_{i}(t)\left(a_{2 i}(t)-b_{2 i i}(t) x_{i}(t)\right), & t \in\left[\tau_{2 k+1}, \tau_{2 k+2}\right), \\
x_{i}\left(\tau_{2 k+2}\right) & =\left(1-D_{2 i}\right) x_{i}\left(\tau_{2 k+2}^{-}\right), \\
& i=1,2, \ldots, n, k=0,1,2, \ldots .
\end{aligned}
$$

Hence, for each $i=1,2, \ldots, n$, we have $x_{i}(t) \leqslant u_{i}(t)$ for all $t \geqslant 0$, where $u_{i}(t)$ is the positive solution of system (34) with initial condition $u_{i}(0)=x_{i}(0)$. According to conditions (56) and (57), by Lemma $1(\mathrm{~b})$, we finally have

$$
\lim _{t \rightarrow \infty} x_{i}(t) \leqslant \lim _{t \rightarrow \infty} u_{i}(t)=0, \quad i=1,2, \ldots, n
$$

for any positive solution $x(t)=\left(x_{1}(t), x_{2}(t), \ldots, x_{n}(t)\right)$ of system (3). Theorem 4 is completed.

\section{Periodic Solutions}

In this section, we study the existence, uniqueness, and the global stability of the positive periodic solution of system (3).

Let $x(t)=\left(x_{1}(t), x_{2}(t), \ldots, x_{n}(t)\right)$ and $x^{*}(t)=$ $\left(x_{1}^{*}(t), x_{2}^{*}(t), \ldots, x_{n}^{*}(t)\right)$ be any two positive solutions of system (3). From Theorem 3, we can obtain that there are constants $A>0$ and $B>0$ such that

$$
\begin{aligned}
& A \leqslant x_{i}(t) \leqslant B, \\
& A \leqslant x_{i}^{*}(t) \leqslant B,
\end{aligned}
$$

$$
\forall t \geqslant 0, i=1,2, \ldots, n \text {. }
$$

Theorem 5. Suppose that all the conditions of Theorem 3 hold and there are constants $c_{i}>0(i=1,2, \ldots, n)$ and $a$ nonnegative continuous function $g(t)$, satisfying

$$
\int_{0}^{\infty} g(t) d t=\infty
$$


such that

$$
\begin{aligned}
& \min \left\{c_{i} b_{1 i i}(t)-\sum_{j \neq i}^{n} c_{j} b_{1 j i}(t), c_{i} b_{2 i i}(t)-\sum_{j \neq i}^{n} c_{j} b_{2 j i}(t)\right\} \\
& \geqslant g(t) \quad \forall t \geqslant 0, i=1,2, \ldots, n .
\end{aligned}
$$

Then system (3) has a unique positive T-periodic solution $x^{*}(t)=\left(x_{1}^{*}(t), x_{2}^{*}(t), \ldots, x_{n}^{*}(t)\right)$ which is globally attractive; that is, any positive solution $x(t)=\left(x_{1}(t), x_{2}(t), \ldots, x_{n}(t)\right)$ of system (3) satisfies

$$
\lim _{t \rightarrow \infty}\left(x_{i}(t)-x_{i}^{*}(t)\right)=0, \quad i=1,2, \ldots, n .
$$

Proof. Choose Lyapunov function as follows:

$$
V(t)=\sum_{i=1}^{n} c_{i}\left|\ln x_{i}(t)-\ln x_{i}^{*}(t)\right|
$$

For any impulsive time $\tau_{k+1}(k=0,1,2, \ldots)$, we have

$$
\begin{aligned}
& V\left(\tau_{k+1}\right) \\
& =\sum_{i=1}^{n} c_{i} \mid \ln \left(\frac{(-1)^{k}+1}{2}\left(1-D_{1 i}\right)+\frac{(-1)^{k+1}+1}{2}\left(1-D_{2 i}\right)\right) \\
& \cdot x_{i}\left(\tau_{k+1}^{-}\right) \\
& \quad-\ln \left(\frac{(-1)^{k}+1}{2}\left(1-D_{1 i}\right)+\frac{(-1)^{k+1}+1}{2}\left(1-D_{2 i}\right)\right) \\
& \quad \cdot x_{i}^{*}\left(\tau_{k+1}^{-}\right)\left|=\sum_{i=1}^{n} c_{i}\right| \ln x_{i}\left(\tau_{k+1}^{-}\right)-\ln x_{i}^{*}\left(\tau_{k+1}^{-}\right) \mid=V\left(\tau_{k+1}^{-}\right) .
\end{aligned}
$$

Hence, $V(t)$ is continuous for all $t \in R_{+}$. On the other hand, from (61) we can obtain that for each $i=1,2, \ldots, n$ and any $t \in R_{+}$and $t \neq \tau_{k+1}$

$$
\begin{aligned}
\frac{1}{B}\left|x_{i}(t)-x_{i}^{*}(t)\right| & \leqslant\left|\ln x_{i}(t)-\ln x_{i}^{*}(t)\right| \\
& \leqslant \frac{1}{A}\left|x_{i}(t)-x_{i}^{*}(t)\right| .
\end{aligned}
$$

For any $t \in R_{+}$and $t \neq \tau_{k+1}(k=0,1,2, \ldots)$, calculating the upper right derivative of $V(t)$, from (63) and (67) we obtain

$$
\begin{aligned}
D^{+} V(t)= & \sum_{i=1}^{n} c_{i} \operatorname{sign}\left(x_{i}(t)-x_{i}^{*}(t)\right)\left(\frac{\dot{x}_{i}(t)}{x_{i}(t)}-\frac{\dot{x}_{i}^{*}(t)}{x_{i}^{*}(t)}\right) \\
& =\left\{\begin{array}{l}
\sum_{i=1}^{n} c_{i} \operatorname{sign}\left(x_{i}(t)-x_{i}^{*}(t)\right)\left(-b_{1 i i}(t)\left(x_{i}(t)-x_{i}^{*}(t)\right)-\sum_{j \neq i}^{n} b_{1 i j}(t)\left(x_{j}(t)-x_{j}^{*}(t)\right)\right), \quad t \in\left[\tau_{2 k}, \tau_{2 k+1}\right), \\
\sum_{i=1}^{n} c_{i} \operatorname{sign}\left(x_{i}(t)-x_{i}^{*}(t)\right)\left(-b_{2 i i}(t)\left(x_{i}(t)-x_{i}^{*}(t)\right)-\sum_{j \neq i}^{n} b_{2 i j}(t)\left(x_{j}(t)-x_{j}^{*}(t)\right)\right), \quad t \in\left[\tau_{2 k+1}, \tau_{2 k+2}\right)
\end{array}\right. \\
& \leqslant\left\{\begin{array}{l}
\sum_{i=1}^{n}\left(-c_{i} b_{1 i i}(t)+\sum_{j \neq i}^{n} c_{j} b_{1 j i}(t)\right)\left|x_{i}(t)-x_{i}^{*}(t)\right|, \quad t \in\left[\tau_{2 k}, \tau_{2 k+1}\right), \\
\sum_{i=1}^{n}\left(-c_{i} b_{2 i i}(t)+\sum_{j \neq i}^{n} c_{j} b_{2 j i}(t)\right)\left|x_{i}(t)-x_{i}^{*}(t)\right|, \quad t \in\left[\tau_{2 k+1}, \tau_{2 k+2}\right)
\end{array}\right. \\
\leqslant & -\sum_{i=1}^{n} \min _{t \in R_{+}}\left\{c_{i} b_{1 i i}(t)-\sum_{j \neq i}^{n} c_{j} b_{1 j i}(t), c_{i} b_{2 i i}(t)-\sum_{j \neq i}^{n} c_{j} b_{2 j i}(t)\right\}\left|x_{i}(t)-x_{i}^{*}(t)\right| \leqslant-\sum_{i=1}^{n} g(t)\left|x_{i}(t)-x_{i}^{*}(t)\right| \\
\leqslant & -\delta g(t) V(t),
\end{aligned}
$$

where $\delta=A / \max _{1 \leqslant i \leqslant n}\left\{c_{i}\right\}>0$. From this, we further have for any $t \geqslant 0$

$$
V(t) \leqslant V(0) \exp \left(-\delta \int_{0}^{t} g(s) d s\right) .
$$

Hence, it follows from (62) that $V(t) \rightarrow 0$ as $t \rightarrow \infty$. Therefore, from (61) we obtain

$$
\lim _{t \rightarrow \infty}\left(x_{i}(t)-x_{i}^{*}(t)\right)=0, \quad i=1,2, \ldots, n .
$$

Now let us consider the sequence $\left(x_{1}^{*}\left(m T, z_{0}\right)\right.$, $\left.x_{2}^{*}\left(m T, z_{0}\right), \ldots, x_{n}^{*}\left(m T, z_{0}\right)\right)=z\left(m T, z_{0}\right)$, where $m=1,2, \ldots$ and $z_{0}=\left(x_{1}^{*}(0), x_{2}^{*}(0), \ldots, x_{n}^{*}(0)\right)$. It is compact in the domain $[A, B]^{n}$ since $A \leqslant x_{i}^{*}(t) \leqslant B$ for all $t \geqslant 0$ and $i=1,2, \ldots, n$. Let $\bar{z}$ be a limit point of this sequence, $\bar{z}=\lim _{n \rightarrow \infty} z\left(m_{n} T, z_{0}\right)$. Then $z(T, \bar{z})=\bar{z}$. 
Indeed, since $z\left(T, z\left(m_{n} T, z_{0}\right)\right)=z\left(m_{n} T, z\left(T, z_{0}\right)\right)$ and $z\left(m_{n} T, z\left(T, z_{0}\right)\right)-z\left(m_{n} T, z_{0}\right) \rightarrow 0$ as $m_{n} \rightarrow \infty$, we get

$$
\begin{aligned}
\|z(T, \bar{z})-\bar{z}\|_{[A, B]^{n}} & \\
\leqslant & \left\|z(T, \bar{z})-z\left(T, z\left(m_{n} T, z_{0}\right)\right)\right\|_{[A, B]^{n}} \\
& +\left\|z\left(T, z\left(m_{n} T, z_{0}\right)\right)-z\left(m_{n} T, z_{0}\right)\right\|_{[A, B]^{n}} \\
& \quad+\left\|z\left(m_{n} T, z_{0}\right)-\bar{z}\right\|_{[A, B]^{n}} \longrightarrow 0, \quad n \longrightarrow \infty .
\end{aligned}
$$

The sequence $z\left(m T, z_{0}\right), m=1,2, \ldots$ has a unique limit point. On the contrary, let the sequence have two limit points $\bar{z}=\lim _{n \rightarrow \infty} z\left(m_{n} T, z_{0}\right)$ and $\widetilde{z}=\lim _{n \rightarrow \infty} z\left(m_{n} T, z_{0}\right)$. Then, taking into account (70) and $\widetilde{z}=z\left(m_{n} T, \widetilde{z}\right)$, we have

$$
\begin{aligned}
\|\bar{z}-\tilde{z}\|_{[A, B]^{n}} \leqslant & \left\|\bar{z}-z\left(m_{n} T, z_{0}\right)\right\|_{[A, B]^{n}} \\
& +\left\|z\left(m_{n} T, z_{0}\right)-\tilde{z}\right\|_{[A, B]^{n}} \longrightarrow 0, \\
& n \longrightarrow \infty,
\end{aligned}
$$

and hence $\bar{z}=\widetilde{z}$. The solution $\left(x_{1}^{*}(t, \bar{z}), x_{2}^{*}(t, \bar{z}), \ldots, x_{n}^{*}(t, \bar{z})\right)$ is the unique periodic solution of system (3). By (70), it is globally attractive. This completes the proof of Theorem 5 .

\section{Numerical Simulation and Discussion}

In this paper, we have investigated a class of two-patch impulsive migration periodic $N$-species Lotka-Volterra competitive system. By means of inequality estimation and Lyapunov functions, we have given the criteria for the permanence, extinction, and existence of the unique globally stable positive periodic solution of system (3).

In order to testify the validity of our results, we consider the following two-patch impulsive migration periodic 2species competitive system:

$$
\begin{aligned}
& \dot{x}_{1}(t) \\
& =x_{1}(t)\left(a_{11}(t)-b_{111}(t) x_{1}(t)-b_{112}(t) x_{2}(t)\right), \\
& \dot{x}_{2}(t) \\
& =x_{2}(t)\left(a_{12}(t)-b_{121}(t) x_{1}(t)-b_{122}(t) x_{2}(t)\right), \\
& x_{1}\left(\tau_{2 k+1}\right)=\left(1-D_{11}\right) x_{1}\left(\tau_{2 k+1}^{-}\right), \\
& x_{2}\left(\tau_{2 k+1}\right)=\left(1-D_{12}\right) x_{2}\left(\tau_{2 k+1}^{-}\right), \\
& \dot{x}_{1}(t) \\
& \quad=x_{1}(t)\left(a_{21}(t)-b_{211}(t) x_{1}(t)-b_{212}(t) x_{2}(t)\right), \\
& \dot{x}_{2}(t) \\
& \quad=x_{2}(t)\left(a_{22}(t)-b_{221}(t) x_{1}(t)-b_{222}(t) x_{2}(t)\right), \\
& t \in\left[\tau_{2 k+1}, \tau_{2 k+2}\right),
\end{aligned}
$$

$$
\begin{aligned}
& x_{1}\left(\tau_{2 k+2}\right)=\left(1-D_{21}\right) x_{1}\left(\tau_{2 k+2}^{-}\right), \\
& x_{2}\left(\tau_{2 k+2}\right)=\left(1-D_{22}\right) x_{2}\left(\tau_{2 k+2}^{-}\right),
\end{aligned}
$$$$
k=0,1,2, \ldots
$$

Corresponding to system (34), two subsystems of system (73) are taken as follows:

$$
\begin{aligned}
\dot{x}_{i}(t) & =x_{i}(t)\left(a_{1 i}(t)-b_{1 i i}(t) x_{i}(t)\right), \\
t & \in\left[\tau_{2 k}, \tau_{2 k+1}\right), \\
x_{i}\left(\tau_{2 k+1}\right) & =\left(1-D_{1 i}\right) x_{i}\left(\tau_{2 k+1}^{-}\right), \\
\dot{x}_{i}(t) & =x_{i}(t)\left(a_{2 i}(t)-b_{2 i i}(t) x_{i}(t)\right), \\
t & \in\left[\tau_{2 k+1}, \tau_{2 k+2}\right), \\
x_{i}\left(\tau_{2 k+2}\right) & =\left(1-D_{2 i}\right) x_{i}\left(\tau_{2 k+2}^{-}\right), \\
i &
\end{aligned}
$$

In system (73), we take $\tau_{0}=0, \tau_{m}-\tau_{m-1}=1$ for all $m=1,2, \ldots$. Hence, we have $T=\tau_{2}=2$. Moreover, we take $D_{11}=D_{12}=0.2, D_{21}=D_{22}=0.3, a_{11}(t)=1+|\sin (2 \pi t)|$, $a_{12}(t)=1.4+0.55 \sin (\pi t), a_{21}(t)=-0.2+|\sin (2 \pi t)|$, $a_{22}(t)=-0.2+0.5 \sin (\pi t), b_{111}(t)=0.2+0.4|\cos (2 \pi t)|$, $b_{112}(t)=0.1|\sin (2 \pi t)|, b_{121}(t)=0.2|\sin (2 \pi t)|, b_{122}(t)=0.55+$ $0.4 \cos (\pi t), b_{211}(t)=1+0.8 \cos (\pi t), b_{212}(t)=0.2+0.1 \sin (\pi t)$, $b_{221}(t)=0.3+0.1 \cos (\pi t), b_{222}(t)=0.3+0.1 \cos (\pi t)$. Obviously,

$$
\begin{aligned}
& \int_{0}^{1} b_{111}(t) d t+\int_{1}^{2} b_{211}(t) d t=1.4546>0 \\
& \int_{0}^{1} a_{11}(t) d t+\int_{1}^{2} a_{21}(t) d t+\ln \left(1-D_{11}\right) \\
& \quad+\ln \left(1-D_{21}\right)=1.4934>0
\end{aligned}
$$

which guarantee that system (74) with $i=1$ has a globally attractively positive 2-periodic solution $x_{1}^{*}(t)$ from Lemma 1(a) and $0.5 \leqslant x_{1}^{*}(t) \leqslant 2$. See Figure 1(a). Similarly, we have

$$
\begin{aligned}
& \int_{0}^{1} b_{122}(t) d t+\int_{1}^{2} b_{222}(t) d t=0.85>0 \\
& \int_{0}^{1} a_{12}(t) d t+\int_{1}^{2} a_{22}(t) d t+\ln \left(1-D_{12}\right) \\
& \quad+\ln \left(1-D_{22}\right)=0.6520>0
\end{aligned}
$$

that is, system (74) with $i=2$ also has a globally attractively positive 2-periodic solution $x_{2}^{*}(t)$ from Lemma $1(\mathrm{a})$ and $0.2 \leq$ $x_{1}^{*}(t) \leq 1.8$. See Figure 1(b). 


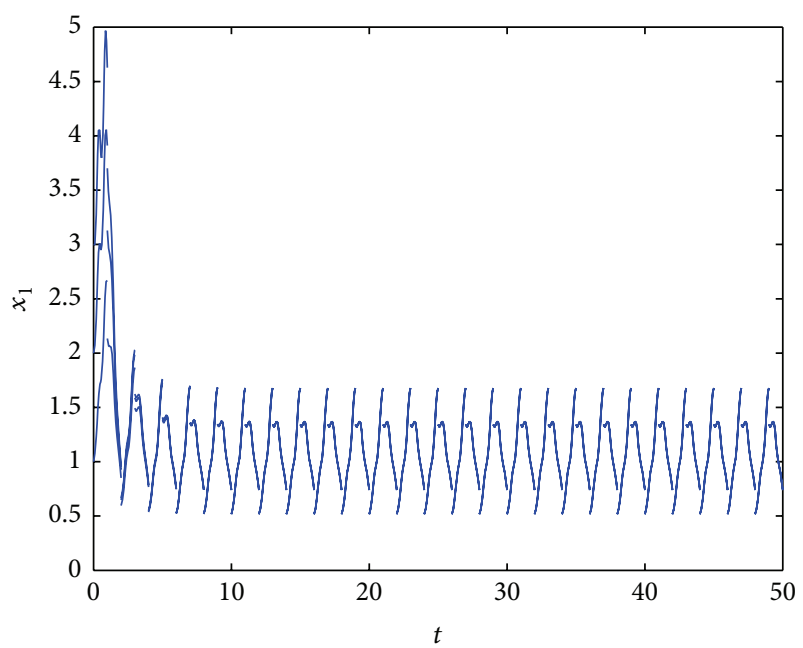

(a)

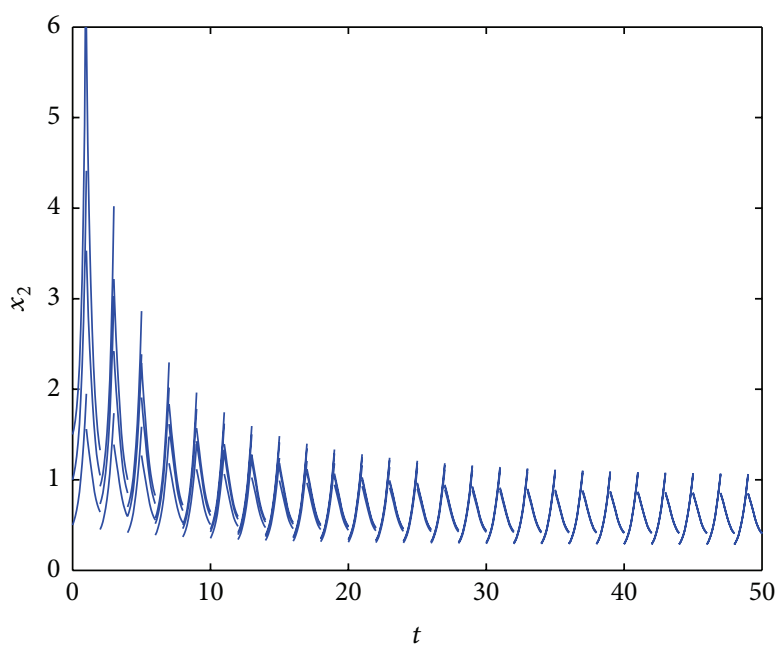

(b)

FIGURE 1: Dynamical behavior of system (74) for $i=1$ (a) and $i=2(\mathrm{~b})$, the unique globally attractively positive 2-periodic solutions $x_{1}^{*}(t)$ and $x_{2}^{*}(t)$. Here, we take initial values $x_{110}=3, x_{120}=2, x_{130}=1, x_{210}=1.5, x_{220}=1$, and $x_{230}=0.5$.

Further, it is not difficult to verify that

$$
\begin{aligned}
& \int_{0}^{\tau_{1}}\left(a_{11}(t)-b_{112}(t) x_{2}^{*}(t)\right) d t \\
& +\int_{\tau_{1}}^{T}\left(a_{21}(t)-b_{212}(t) x_{2}^{*}(t)\right) d t+\ln \left(1-D_{11}\right) \\
& +\ln \left(1-D_{21}\right) \geqslant \int_{0}^{\tau_{1}}\left(a_{11}(t)-1.8 b_{112}(t)\right) d t \\
& +\int_{\tau_{1}}^{T}\left(a_{21}(t)-1.8 b_{212}(t)\right) d t+\ln \left(1-D_{11}\right) \\
& +\ln \left(1-D_{21}\right)=1.1334>0, \\
& \int_{0}^{\tau_{1}}\left(a_{12}(t)-b_{121}(t) x_{1}^{*}(t)\right) d t \\
& +\int_{\tau_{1}}^{T}\left(a_{22}(t)-b_{221}(t) x_{1}^{*}(t)\right) d t+\ln \left(1-D_{12}\right) \\
& \quad+\ln \left(1-D_{22}\right) \geqslant \int_{0}^{\tau_{1}}\left(a_{12}(t)-2 b_{121}(t)\right) d t \\
& +\int_{\tau_{1}}^{T}\left(a_{22}(t)-2 b_{221}(t)\right) d t+\ln \left(1-D_{12}\right) \\
& +\ln \left(1-D_{22}\right)=0.0974>0,
\end{aligned}
$$

Figure 2: Dynamical behavior of system (73). Obviously, species $x_{1}$ and $x_{2}$ are permanent and 2-periodic. Here, we take initial values $x_{10}=1$ and $x_{20}=2$.

Meanwhile, if we choose $c_{1}=c_{2}=1$ in Theorem 5, we can verify that

$$
\begin{aligned}
\min & \left\{c_{1} b_{111}(t)-c_{2} b_{121}(t), c_{1} b_{211}(t)\right. \\
& -c_{2} b_{221}(t), c_{2} b_{122}(t)-c_{1} b_{112}(t), c_{2} b_{222}(t) \\
& \left.-c_{1} b_{212}(t)\right\} \geqslant 0.05|\cos (2 \pi t)| \geqslant 0 ;
\end{aligned}
$$

then we can choose $g(t)=0.05|\cos (2 \pi t)|$. Therefore we have that $g(t)$ is nonnegative and continuous and $\int_{0}^{\infty} g(t)=$ $\int_{0}^{\infty} 0.05|\cos (2 \pi t)|=\infty$. All conditions of Theorem 5 are which satisfy condition (36) of Theorem 3 for each $i=1,2$. Therefore, species $x_{1}$ and $x_{2}$ are permanent. The numerical simulation is given in Figure 2.

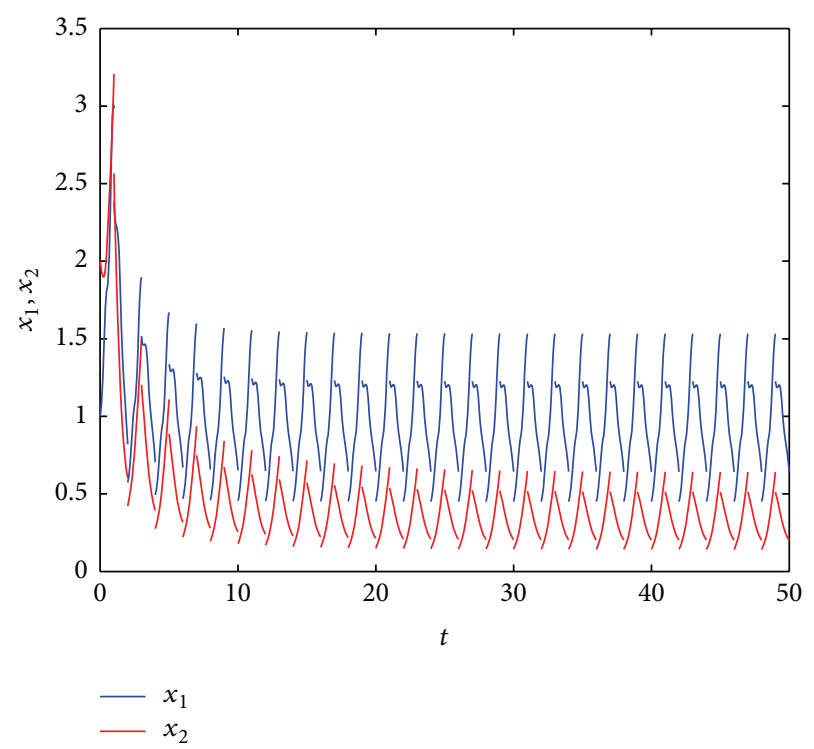

$\int_{0}^{\infty} 0.05|\cos (2 \pi t)|=\infty$. All conditions of Theorem 5 are 


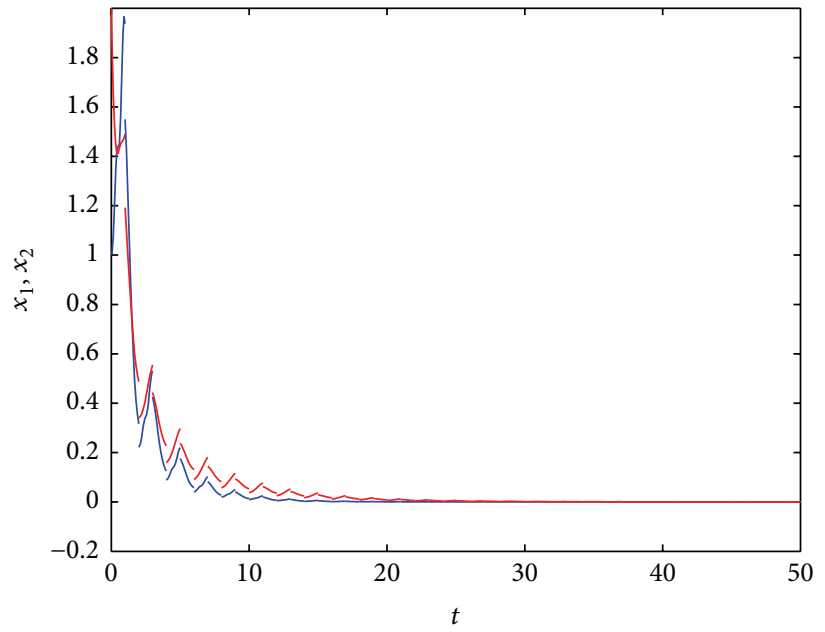

(a)

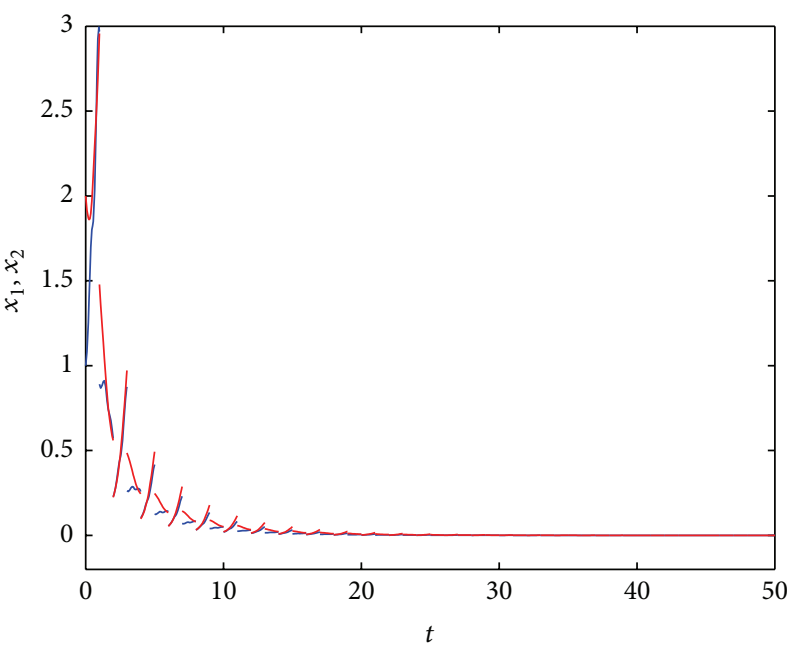

(b)

Figure 3: The extinction of the two species $x_{1}$ and $x_{2}$ of system (73). The extinction illustrated in (a) is caused by the austere survival environment of the two patches and the extinction illustrated in (b) is caused by the large loss during the migration $\left(D_{11}=0.7, D_{12}=0.6\right.$, $D_{21}=0.5$, and $D_{22}=0.6$ ). Here, we take initial values $x_{10}=1$ and $x_{20}=2$.

satisfied. Hence, system (74) has a unique positive 2-periodic solution $\left(\bar{x}_{1}, \bar{x}_{2}\right)$. See also Figure 2 .

However, if the survival environment of the two patches is austere, the intrinsic growth rates of the two species will decrease. Hence, if we take $a_{11}(t)=0.5+|\sin (2 \pi t)|, a_{21}(t)=$ $-0.2+\sin (\pi t), a_{12}(t)=0.4+0.55 \sin (\pi t)$, and $a_{22}(t)=$ $-0.2+0.5 \sin (\pi t)$ and all other parameters are retained, then we obtain

$$
\begin{aligned}
& \int_{0}^{1} a_{11}(t) d t+\int_{1}^{2} a_{21}(t) d t+\ln \left(1-D_{11}\right) \\
& \quad+\ln \left(1-D_{21}\right)=-0.2798<0 \\
& \int_{0}^{1} a_{12}(t) d t+\int_{1}^{2} a_{22}(t) d t+\ln \left(1-D_{12}\right) \\
& \quad+\ln \left(1-D_{22}\right)=-0.3480<0,
\end{aligned}
$$

which satisfy condition (57) of Theorem 4 (condition (56) is obvious). Hence, all species of system (73) will go extinct. See Figure 3(a).

In addition, if the environment of the two patches is survivable, but the migration loss of the two species is large, that is, if we take $D_{11}=0.7, D_{12}=0.6, D_{21}=0.5$, and $D_{22}=0.6$ and all other coefficients are unchanged, then we can verify that

$$
\begin{aligned}
& \int_{0}^{1} a_{11}(t) d t+\int_{1}^{2} a_{21}(t) d t+\ln \left(1-D_{11}\right) \\
& \quad+\ln \left(1-D_{21}\right)=-0.0470<0 \\
& \int_{0}^{1} a_{12}(t) d t+\int_{1}^{2} a_{22}(t) d t+\ln \left(1-D_{12}\right) \\
& \quad+\ln \left(1-D_{22}\right)=-0.3776<0
\end{aligned}
$$

and condition (57) of Theorem 4 satisfies. Therefore, the two species $x_{1}$ and $x_{2}$ of system (73) will also go extinct. See Figure 3(b). Meanwhile, if we fix $a_{11}(t)=1+$ $|\sin (2 \pi t)|, a_{12}(t)=1.4+0.55 \sin (\pi t), a_{21}(t)=-0.2+$ $|\sin (2 \pi t)|$, and $a_{22}(t)=-0.2+0.5 \sin (\pi t)$, let $D_{11}=D_{21}=D_{1}$ and $D_{12}=D_{22}=D_{2}$ denote

$$
\begin{aligned}
f\left(D_{1}\right)= & \int_{0}^{1} a_{11}(t) d t+\int_{1}^{2} a_{21}(t) d t+\ln \left(1-D_{1}\right) \\
& +\ln \left(1-D_{1}\right), \\
g\left(D_{2}\right)= & \int_{0}^{1} a_{12}(t) d t+\int_{1}^{2} a_{22}(t) d t+\ln \left(1-D_{2}\right) \\
& +\ln \left(1-D_{2}\right),
\end{aligned}
$$

and then we have $f\left(D_{1}\right) \leqslant 0$ if $0.6453 \leqslant D_{1}<1$ and $g\left(D_{2}\right) \leqslant$ 0 if $0.4598 \leqslant D_{2}<1$ (see Figure 4); that is, species $x_{1}$ and $x_{2}$ will go extinct if $0.6453 \leqslant D_{1}<1$ and $0.4598 \leqslant D_{2}<1$. This shows that the migration loss during the migration also plays a crucial role on the permanence and extinction of the two species.

Remark 6. In the course of the above discussion, we have established conditions that guarantee that the two species are permanent or extinct simultaneously. Hence, an interesting and important open problem is under what conditions one species is permanent and the other is extinct.

Remark 7. In all of the above discussion, we have established that if

$$
\begin{aligned}
\gamma_{1}= & \int_{0}^{\tau_{1}}\left(a_{11}(t)-b_{112}(t) x_{2}^{*}(t)\right) d t \\
& +\int_{\tau_{1}}^{T}\left(a_{21}(t)-b_{212}(t) x_{2}^{*}(t)\right) d t+\ln \left(1-D_{11}\right) \\
& +\ln \left(1-D_{21}\right)>0,
\end{aligned}
$$




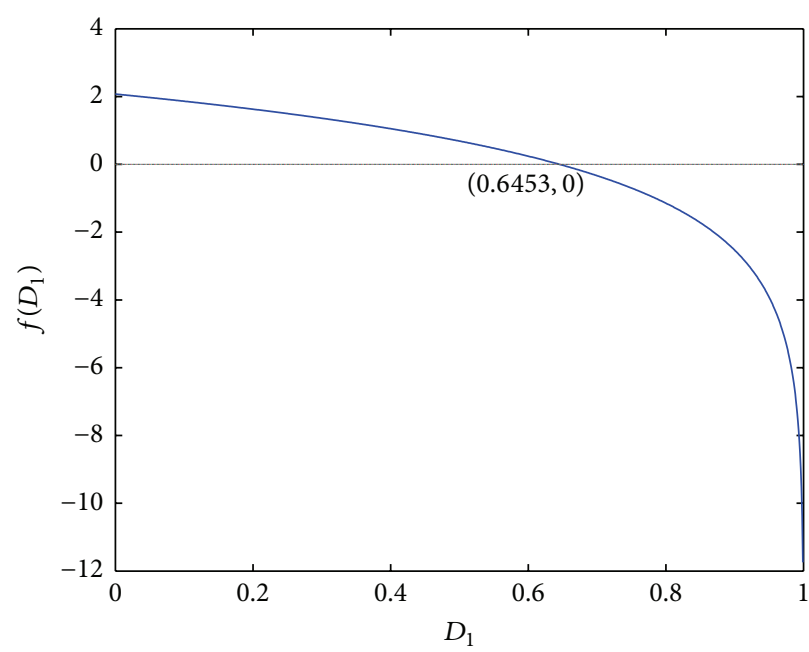

(a)

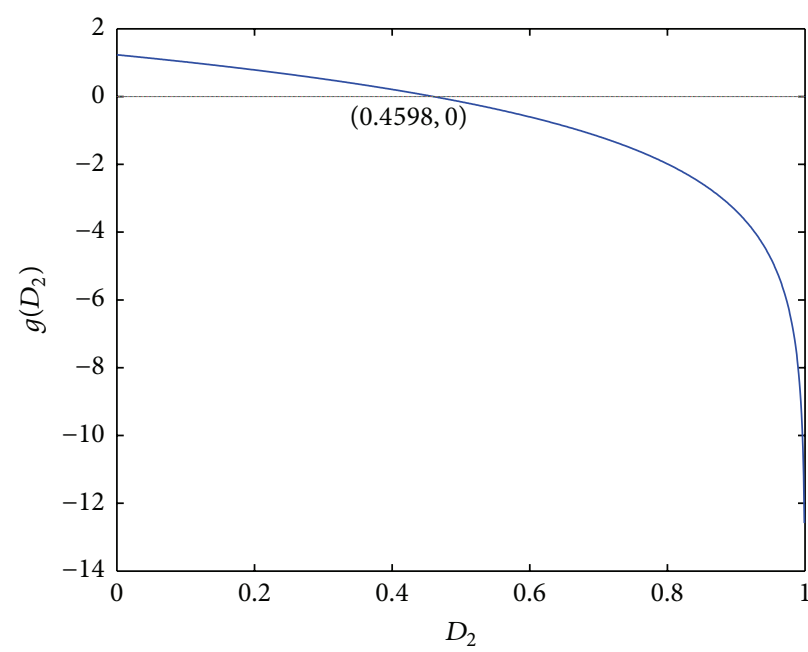

(b)

Figure 4: The trends of $f\left(D_{1}\right)$ on $D_{1}(\mathrm{a})$ and $g\left(D_{2}\right)$ on $D_{2}(\mathrm{~b})$. Obviously, $f\left(D_{1}\right) \leqslant 0$ if $0.6453 \leqslant D_{1}<1$ and $g\left(D_{2}\right) \leqslant 0$ if $0.4598 \leqslant D_{2}<1$.

$$
\begin{aligned}
\gamma_{2}= & \int_{0}^{\tau_{1}}\left(a_{12}(t)-b_{121}(t) x_{1}^{*}(t)\right) d t \\
& +\int_{\tau_{1}}^{T}\left(a_{22}(t)-b_{221}(t) x_{1}^{*}(t)\right) d t+\ln \left(1-D_{12}\right) \\
& +\ln \left(1-D_{22}\right)>0
\end{aligned}
$$

the two species are permanent. And if

$$
\begin{aligned}
\eta_{1}= & \int_{0}^{\tau_{1}} a_{11}(t) d t+\int_{\tau_{1}}^{T} a_{21}(t) d t+\ln \left(1-D_{11}\right) \\
& +\ln \left(1-D_{21}\right) \leqslant 0, \\
\eta_{2}= & \int_{0}^{\tau_{1}} a_{12}(t) d t+\int_{\tau_{1}}^{T} a_{22}(t) d t+\ln \left(1-D_{12}\right) \\
& +\ln \left(1-D_{22}\right) \leqslant 0,
\end{aligned}
$$

then the two species are extinct. Therefore, we have the following open problems.

(O1) If $\gamma_{1}<0<\eta_{1}, \gamma_{2}>0$, and $\eta_{2} \leqslant 0$, what trends of all solutions of system (73) are.

(O2) If $\gamma_{2}<0<\eta_{2}, \gamma_{1}>0$, and $\eta_{1} \leqslant 0$, what trends of all solutions of system (73) are.

(O3) If $\gamma_{1}<0<\eta_{1}$ and $\gamma_{2}<0<\eta_{2}$, what trends of all solutions of system (73) are.

Moreover, if we extend the two-species competitive system to our investigated $N$-species competitive system, what results can be obtained under the similar cases, which are also interesting open problems.

\section{Conflict of Interests}

The authors declare that there is no conflict of interests regarding the publication of this paper.

\section{Acknowledgments}

This research was supported by the National Natural Science Foundation of China (11401060) and Zhejiang Provincial Natural Science Foundation of China (LQ13A010023).

\section{References}

[1] E. Beretta and Y. Takeuchi, "Global asymptotic stability of Lotka-Volterra diffusion models with continuous time delay," SIAM Journal on Applied Mathematics, vol. 48, no. 3, pp. 627651, 1988.

[2] Z. Y. Lu and Y. Takeuchi, "Permanence and global stability for cooperative Lotka-Volterra diffusion systems," Nonlinear Analysis. Theory, Methods \& Applications, vol. 19, no. 10, pp. 963975, 1992.

[3] Z. Teng and Z. Lu, "The effect of dispersal on single-species nonautonomous dispersal models with delays," Journal of Mathematical Biology, vol. 42, no. 5, pp. 439-454, 2001.

[4] J. Cui, "Dispersal permanence of a periodic predator-prey system with Beddington-DeAngelis functional response," Nonlinear Analysis: Theory, Methods \& Applications, vol. 64, no. 3, pp. 440-456, 2006.

[5] L. Zhang and Z. Teng, "Boundedness and permanence in a class of periodic time-dependent predator-prey system with prey dispersal and predator density-independence," Chaos, Solitons and Fractals, vol. 36, no. 3, pp. 729-739, 2008.

[6] Z. Liu and S. Zhong, "Permanence and extinction analysis for a delayed periodic predator-prey system with Holling type II response function and diffusion," Applied Mathematics and Computation, vol. 216, no. 10, pp. 3002-3015, 2010.

[7] L. Wang and Z. Liu, "Impulsive diffusion in single species model," Chaos, Solitons and Fractals, vol. 33, no. 4, pp. 1213-1219, 2007.

[8] J. Jiao, L. Chen, S. Cai, and L. Wang, "Dynamics of a stagestructured predator-prey model with prey impulsively diffusing between two patches," Nonlinear Analysis: Real World Applications, vol. 11, no. 4, pp. 2748-2756, 2010. 
[9] J. Jiao, X. Yang, S. Cai, and L. Chen, "Dynamical analysis of a delayed predator-prey model with impulsive diffusion between two patches," Mathematics and Computers in Simulation, vol. 80, no. 3, pp. 522-532, 2009.

[10] Y. Shao, "Analysis of a delayed predator-prey system with impulsive diffusion between two patches," Mathematical and Computer Modelling, vol. 52, no. 1-2, pp. 120-127, 2010.

[11] L. Zhang, Z. Teng, D. L. DeAngelis, and S. Ruan, "Single species models with logistic growth and dissymmetric impulse dispersal," Mathematical Biosciences, vol. 241, no. 2, pp. 188-197, 2013.

[12] Z. Liu, Z. Teng, and L. Zhang, "Two patches impulsive diffusion periodic single-species logistic model," International Journal of Biomathematics, vol. 3, no. 1, pp. 127-141, 2010.

[13] Z. Liu, S. Zhong, C. Yin, and W. Chen, "Two-patches prey impulsive diffusion periodic predator-prey model," Communications in Nonlinear Science and Numerical Simulation, vol. 16, no. 6, pp. 2641-2655, 2011.

[14] J. Hou, Z. Teng, and S. Gao, "Permanence and global stability for nonautonomous $N$-species Lotka-Valterra competitive system with impulses," Nonlinear Analysis: Real World Applications, vol. 11, no. 3, pp. 1882-1896, 2010. 


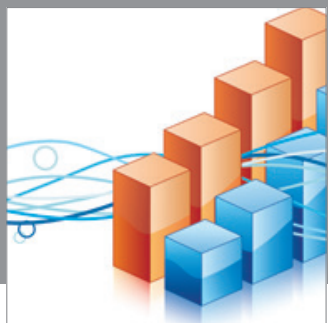

Advances in

Operations Research

mansans

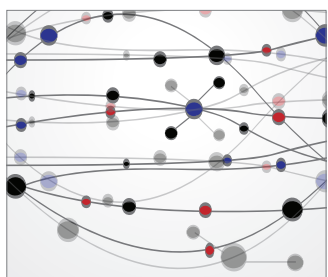

The Scientific World Journal
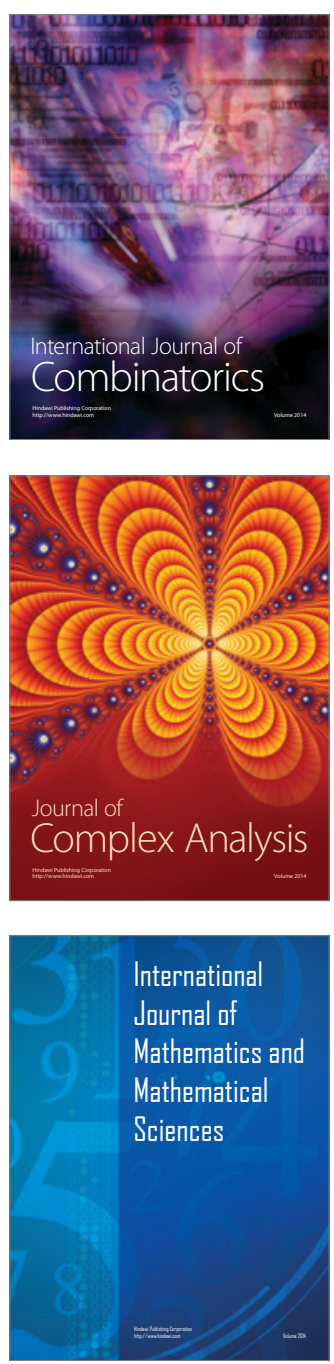
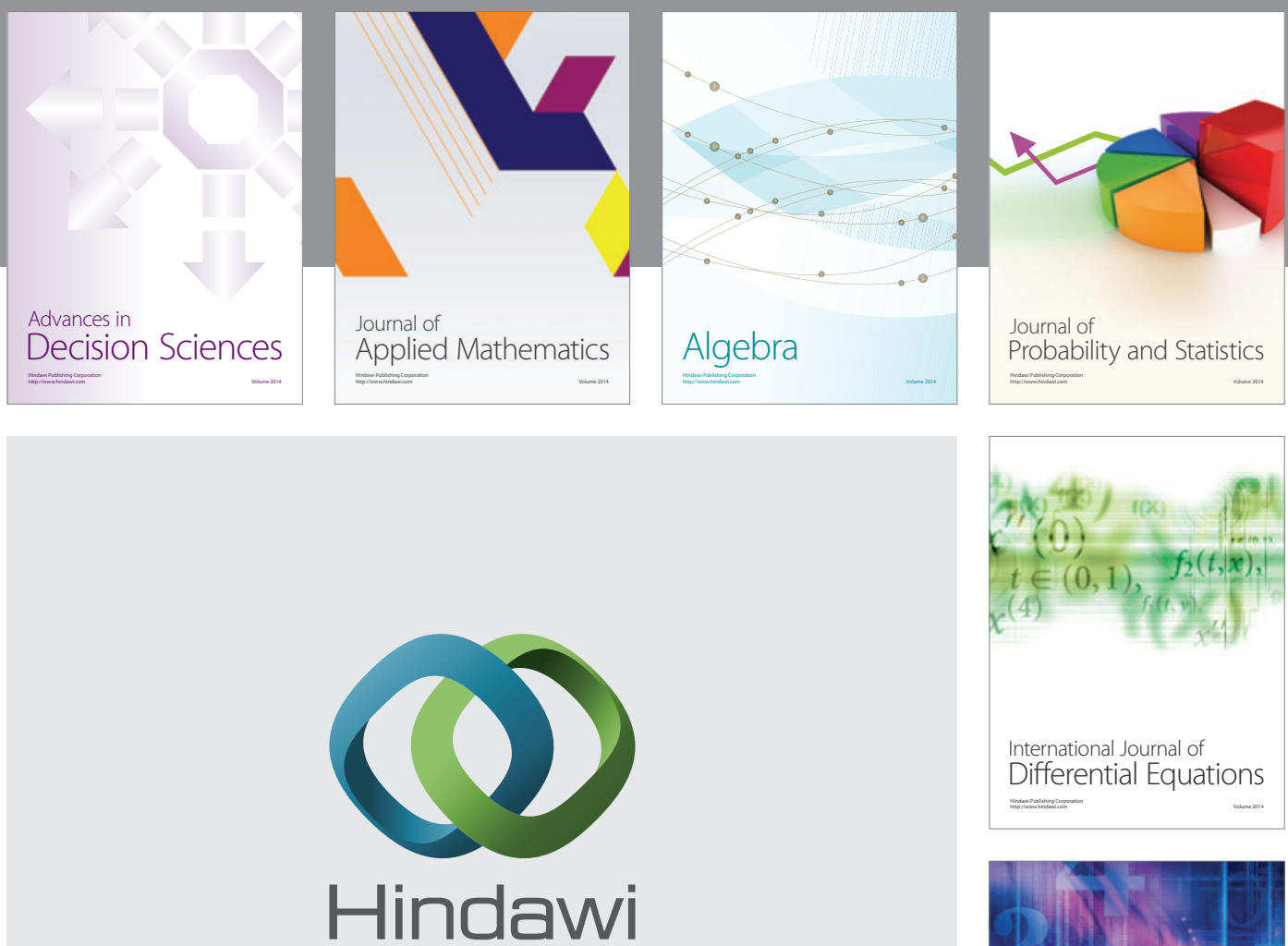

Submit your manuscripts at http://www.hindawi.com
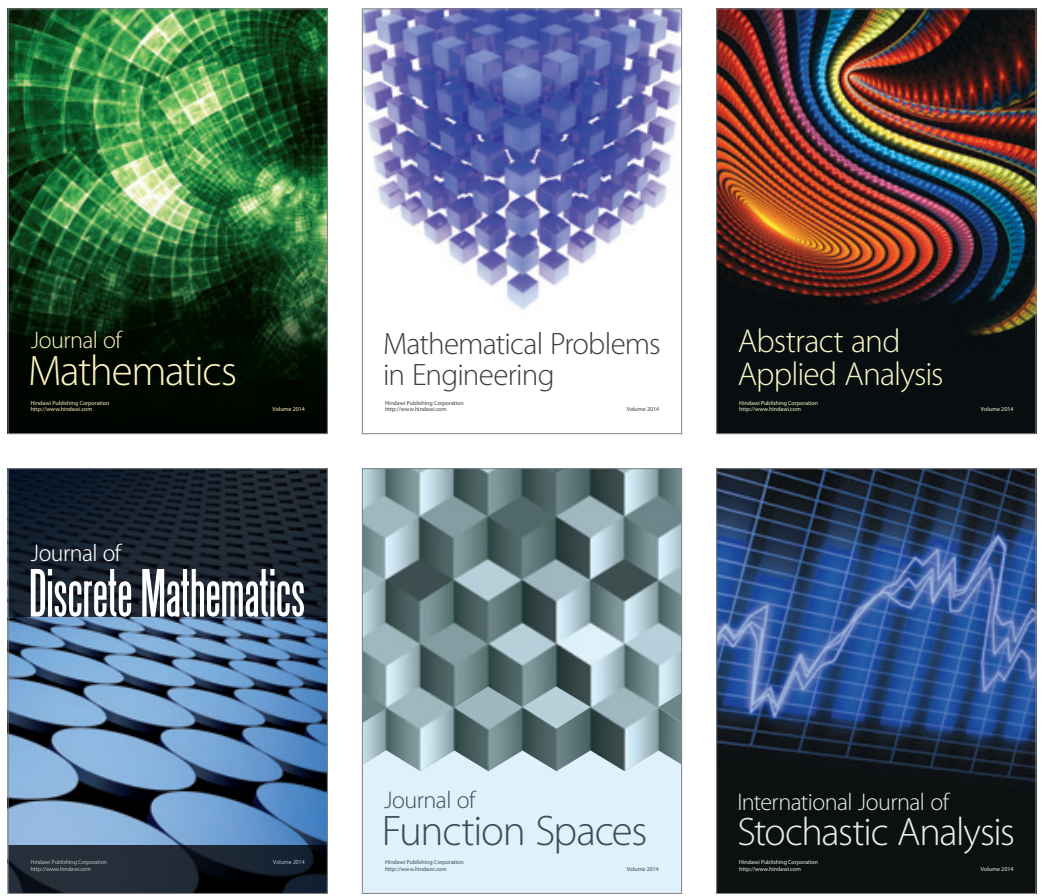

Journal of

Function Spaces

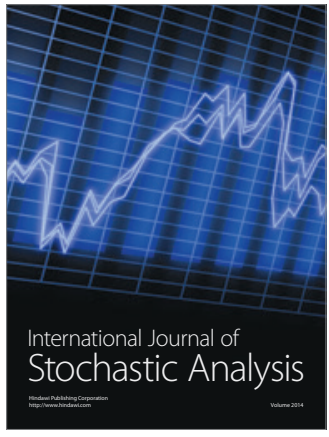

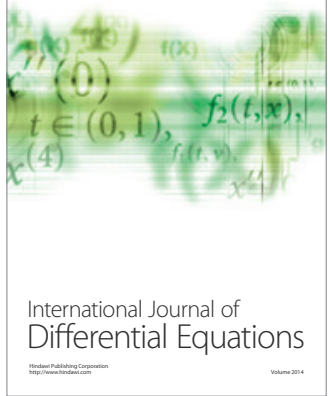
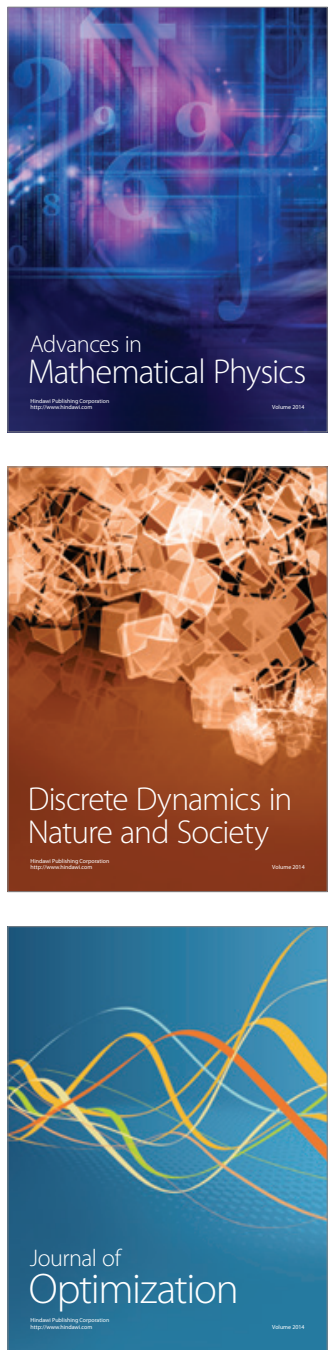